\title{
Antimicrobial Resistance Patterns of Selected Enterobacteriaceae Isolated from Commercial Poultry Production Systems in Kiambu County, Kenya
}

\author{
James Gakunga Ndukui1,2*, Joseph Kangangi Gikunju³, Gabriel Oluga Aboge, John Mwaniki, \\ Samuel Kariuki ${ }^{4}$, James Mucunu Mbaria1 \\ ${ }^{1}$ Department of Public Health, Pharmacology and Toxicology, College of Agriculture and Veterinary Sciences, \\ University of Nairobi, Kenya \\ ${ }^{2}$ Department of Nursing, Catholic University of Eastern Africa, Langata Campus, Nairobi, Kenya \\ ${ }^{3}$ Department of Medical Laboratory Sciences, College of Health Science, Jomo Kenyatta University of Agriculture and Technology, \\ Nairobi, Kenya \\ ${ }^{4}$ Centre for Microbiology Research, Kenya Medical Research Centre, Nairobi, Kenya \\ Email: *ndukuiga@gmail.com, *jndukui@students.uonbi.ac.ke
}

How to cite this paper: Ndukui, J.G., Gikunju, J.K., Aboge, G.O., Mwaniki, J., Kariuki, S. and Mbaria, J.M. (2021) Antimicrobial Resistance Patterns of Selected Enterobacteriaceae Isolated from Commercial Poultry Production Systems in Kiambu County, Kenya. Pharmacology \& Pharmacy, 12, 219-236.

https://doi.org/10.4236/pp.2021.1210019

Received: August 12, 2021

Accepted: October 22, 2021

Published: October 25, 2021

Copyright $\odot 2021$ by author(s) and Scientific Research Publishing Inc. This work is licensed under the Creative Commons Attribution International License (CC BY 4.0).

http://creativecommons.org/licenses/by/4.0/ (c) (i) Open Access

\begin{abstract}
Introduction: In the last two decades, the treatment of enteric infections has been complicated by the emergence of antimicrobial resistant strains. Occurrence of multidrug resistant Extended Spectrum Beta Lactamase (ESBL) producing Enterobactaeraceae poses the greatest risk to public health by raising morbidity and mortality by six folds in developing countries. The present study aims to determine the antibiotics resistance patterns of selected Enterobacteriaceae isolated from commercial poultry production systems in Kiambu County. Methods: A laboratory based cross-sectional study was conducted in six purposively selected Sub-Counties of Kiambu County from October 2020, to February 2021. A total of 437 fecal samples were collected from each household. The antibiotic susceptibility testing using disk diffusion method was used against E. coli; Salmonella spps.; Shigella spps.; and Klebsiella spps. which were isolated and identified through standard biochemical. Results: Out of 437 fecal and stool samples collected, 591 isolates were recovered with E. coli (48.9\%) being the most frequently identified, followed by Shigella spps. (18.8\%), Salmonella spps. (18.3\%), and Klebsiella spps. (14.0\%). The study shows there was high prevalence of multiple resistance among isolates especially to Sulfamethoxazole (79\%), Trimethoprim (71\%), and Tetracyclines (59\%), correspondingly. Additionally, the isolates showed the highest rate of susceptibility against Cefuroxime (94\%), Gentamicin (93\%), Ceftriaxone (91\%),
\end{abstract}


Cefepime (89\%), Cefotaxime (85\%), Ceftazidime (84\%), and Chloramphenicol $(77 \%)$, respectively. Discussion: Our study indicates that both fecal and stool materials from commercial poultry and humans can be reservoir of multi-drug resistance enteric's which can be a potential route of transmission of resistance genes, which pose a great risk to public health of Kiambu Residence.

\section{Keywords}

Antimicrobial Resistance, Enterobacteraceae, AST, Commercial Poultry Production, Multi-Drug Resistance

\section{Introduction}

In the last two decades, the treatment of enteric disease in humans and poultry has been complicated by the emergence of antimicrobial resistant pathogens. Emerging antimicrobial resistant extended spectrum beta Lactamase (ESBL) producing Enterobacteriaceae is a serious threat to public health [1]. The rapid increase in the prevalence of ESBL producing Gram-negative pathogens that are resistant to fluoroquinolones, aminoglycosides, $\beta$-lactams and colistin has been rarely reported [2] [3] [4]. Other resistance mechanisms against beta-lactams are the outer membrane permeability change and efflux pumps. The over and mis-use of antimicrobials agents in livestock lead to development of resistance and subsequent transfer of resistance genes among bacteria to animals, animal products and the environment [5] [6] [7]. On the other hand, judicious use of antimicrobial agents is required, but acceptable strategies to achieve this goal and to address the challenges must be devised and communicated [8]. Antimicrobial resistance is a stark reality across the globe, including Kenya. The challenges associated with controlling antibiotic resistance, particularly in Kenya, are multifaceted.

Commercial poultry industry is well developed and it is the largest supplier of animal protein in form of meat and eggs worldwide. Poultry is relatively cheap and is kept in small areas basically providing income and protein to the families [9] [10]. However, poultry diseases are some of the limiting factors to this industry. The increased poultry disease burden has accentuated the high demand and the use of veterinary antimicrobial drugs [11].

Majority of Kenyans depend on poultry products for livelihood and survival, therefore low production levels affect their income levels. This affects their contribution to the country's GDP and global economic development, halting the achievement of the Medium term plan 111 as set by the government on sustainable development through agriculture. In Kenya, poultry production is one of the fastest means of ameliorating the animal protein deficiency. The high turn-over rate and the quest for white meat have given more credence to poultry farming. The need to meet the demand for white meat has necessitated the large scale 
production and subsequent use of antimicrobial agents as growth promoters. Presence of drugs or antimicrobial residues in food is recognized worldwide by various public health authorities as being illegal [12], and their consumption could result in public health hazards including: development of resistant strains of microorganisms, respiratory infections, cancer, carcinogenicity, hypersensitive reaction in sensitized individuals [13], and distortion of intestinal microflora [14] [15] [16], and (European Centre for Disease Prevention and Control (ECDC), [17].

The looming antibiotic-resistance crisis has penetrated the consciousness of clinicians, researchers, policymakers, politicians and the public at large. The evolution and widespread distribution of ESBL producing bacterial pathogens have made diseases that were once easily treatable deadly again. Unfortunately, accompanying the rise in global resistance is a failure in antibacterial drug discovery. Lessons from the history of antibiotic discovery and fresh understanding of antibiotic action and the cell biology of microorganisms have the potential to deliver twenty-first century medicines that are able to control infection in the resistance era [18]. The usage of antimicrobial drugs in poultry production is very important in disease treatment, prevention and growth promoting but its use must be accepted as a responsibility rather than a right when trying to improve poultry health [5]. This will help to minimize the potential risk and hazards due to poor drug use of antimicrobial agents in livestock production. Therefore, use of veterinary antibiotic drugs in poultry systems needs proper control through legislation bodies with broad goal to preserve animal health, improve animal production and protect the public [19]. Therefore, the study aims to determine the antimicrobial resistance patterns of selected Enterobacteriaceae isolated from commercial poultry production systems and humans in Kiambu County, Kenya.

\section{Materials and Methods}

\subsection{Study Area}

Kiambu County covers an area of $13,191 \mathrm{~km}^{2}$ and is located to north of Nairobi and west of Mt. Kenya and it's estimated to have 4,383,743 inhabitants according to 2009 population census. The high population growth in this area, favors commercial poultry production more than other types of livestock production. This is because Kiambu is a predominantly an intensive small holder production region, it pattern and extend of antimicrobial use significantly differs from other regions of Kenya. According to the report by Nyaga [20], Kiambu County also has got 5351 (broiler farmers) and 1185 (layer farmers) who are for commercial purposes and 12,633 for dual purpose activity.

\subsection{Study Population}

The current study targeted farmers practicing Commercial poultry production systems in Kiambu County and therefore keeping Broiler, layers and Improved 
Kienyeji birds for business.

\subsection{Study Design}

A laboratory based cross-sectional study was carried out from November 2020, to February 2021 in six purposively selected sub-counties namely; Juja, Ruiru, Thika, Gatundu South, Gatundu north and Kikuyu in Kiambu County, Kenya. Four hundred and Thirty seven (437) cloacal swabs (Broiler, layers and Improved Kienyeji) and stool samples from the farmers were collected and submitted to the Centre for Microbiology Research-KEMRI for analysis. From this samples 591 isolates of target enterobacteriaceae (E. coli; Salmonell spps.; Shigella spps. and Klebsiella spps.) were identified.

\subsection{Sample Size Estimation}

The sample size was calculated according to [21]. Using this formula, the sample size calculated was estimated to be 400 fecal samples at CI of 95\%. The fecal samples were distributed among farmers and chicken (layers; broilers; improved Kienyeji).

$$
n=\frac{t^{2} * p(1-p)}{m^{2}}
$$

where; $P=$ estimated prevalence, $M=$ Margin of errors (Standard deviation of 0.05), $T=$ Confidence level at 95\% (Standard deviations of 1.96). $n=$ required sample size. $n \approx 400$ approximated fecal samples. This fecal samples were distributed among Farmers and poultry (broiler; layers; Kienyeji).

\subsection{Sample Collection}

Four hundred and thirty seven $(n=437)$ fecal and stool samples were collected from the six purposively selected representative sub-counties in Kiambu County. Approximately $5 \mathrm{~g}$ of fresh fecal samples was aseptically collected from Humans $(n=72)$, and chicken cloacal swabs $(n=365)$ was collected for this study from the various farms on healthy birds that was first to come out of the animal house. Each fecal and cloacal swabs was placed in Amies and SF transport media, stored in cool box and transported to the Centre of Microbiology Research (CMR)-KEMRI, within 6 hours. Upon arrival to the lab the samples were incubated at $37^{\circ} \mathrm{C}$ overnight for 18 to 24 hours. Thereafter removal from the incubator the plates were labelled as the MAC and XLD/SS respectively. The incubated Amies and Selenite F (SF) samples were mixed uniformly. A sterile wire loop was used to pick a loopful mixture separately from both Amies and SF with streaking on MAC and SS agar differential culture plates to form distinct colonies. The plates were incubated at $37^{\circ} \mathrm{C}$ overnight for 18 to 24 hours, followed by reading to identify the enteric's of interest (E. coli, Klebsiella Pneumonia spps., Salmonella spps. and Shigella spps.) using morphological characteristics. The morphologically identified isolates were stocked in tryptic soy broth and kept in $-20^{\circ} \mathrm{C}$ refrigerator awaiting for biochemical identification, antibiotic susceptibil- 
ity testing (AST) and molecular genotyping.

\subsection{Biochemical Identification of the Isolates}

Pure colonies were selected and sub-cultured on nutrient agar slant and were further processed for biochemical tests. Well-known traditional biochemical tests and selective media for bacteria identification were employed [22]. The isolates were identified using MacConkey broth (Sigma-Aldrich), and Simmons citrate agar (Sigma_Aldrich), catalase, sugar fermentation (Triple sugar iron (TSI) agar), indole, citrate utilization, urease production, and motility tests. The identified bacterial species were kept in a $20 \%$ glycerol/medium mixture at $-80^{\circ} \mathrm{C}$ at the Molecular and microbiology laboratory of Centre for Microbiology Research-KEMRI.

\subsection{Bacterial Isolation}

A total of 591 isolates of Escherichia coli $(n=289)$; Klebseilla pneumonia ( $n=$ 83); Salmonella spps. $(n=108)$ and Shigella spps. $(n=111)$ were isolated from stool and fecal obtained from farmers practicing commercial poultry production in Kiambu County-Kenya from November, 2020 to February, 2021. All the isolates were collected under approved ethical standards as per Clinical and Laboratory standard institute guidelines [23]. The isolates were identified and verified using standard biochemical reactions including: growth on MacConkey agar, citrate utilization, Voges Proskauer, methyl Red, and motility tests as per Amira Mohamed EL-Ganiny et al. [24]; Koneman et al. [25].

\subsection{Antibiotic Susceptibility Tests}

The antibiotic resistance pattern of isolates form E. coli; Shigella spps.; Salmonella spps. and Klebsiella spps. was determined using the disk diffusion method on Muller-Hinton agar according to the Clinical and Laboratory Standard Institute guidelines [23] [26]. The antibiotic discs were obtained from Oxiod (USA). Thirteen antibiotics were tested and distributed among plate A and B. Plate A had the following: Amoxacillin-Clavullanic acid (AMC, $10 \mu \mathrm{g}$ ); Ampicillin (AMP, $10 \mu \mathrm{g}$ ); Ceftriaxone (CRO); Cefiximine (CFM); Cefotaxime (CTX); Cefepime (FEP); Ceftazidime-Cefodoxime (CAZ/CPD) and plate B had the following: Ciprofloxacin (CIP, $5 \mu \mathrm{g}$ ); Trimethoprim (TMP); Gentamicin (GEN, $10 \mu \mathrm{g}$ ); Tetracycline (TET, $100 \mu \mathrm{g}$ ); Sulfamethoxazole (SMX, $200 \mu \mathrm{g}$ ); Chloramphenicol (CHL, $30 \mu \mathrm{g}$ ); Ceftazidime (CAZ). The dried Mueller Hinton agar plate was plated with the isolated microbes. The correct antimicrobial impregnated disks were placed on the surface of the agar using sterilized forceps and incubated at $35^{\circ} \mathrm{C}$ overnight. The antibiotic inhibition zones were measured to the nearest millimeter $(\mathrm{mm})$ from the center of the disk to a point on the circumference of the zone where a distinct edge was seen using a rule. For all isolates the zones of inhibition were determined and interpreted as per breakpoints. Phenotypic detection of ESBLs production was detected by a double-disk synergy test (DDST). Enhancement of the inhibition zone between the disks containing Amoxacil- 
lin-Clavullanic acid (AMC) and Cefotaxime or Ceftazidime indicated presence of ESBL production.

\subsection{Quality Controls}

The quality control was run using E. coli ATCC25922 and K. Pneumoniae ATCC700603. The cultures were classified was sensitive, intermediate and resistance on the basis of diameter of zones of inhibition. Isolates resistant to three or more antimicrobial were considered Multi-resistance [27].

\subsection{Data Analysis and Presentation}

The inhibition zone diameter were measured by use of divider and a ruler, and the mean, and standard errors of three replicates calculated, analyzed and keyed into EPICOLLECT with results presented in form of tables and graphs

\section{Results}

Table 1 presents results of total selected isolates of enterobacteriaceae recovered from commercial poultry and humans in Kiambu County. Out of 437 fecal and stool samples collected 591 isolates recovered E. coli (48.9\%) were the most frequently identified, while seconded by Shigella spps. (18.8\%), Salmonella spps. with least number being Klebsiella spps. (14.0\%) as shown in Table 1 below.

Table 2 presents results of antibiotics resistance profiles of 591 Enterobacteriaceae isolates against to 13 antibiotics. The results indicate that there is multi-drug resistance across the various classes of antibiotics used among the four selected enterobacteriaceae. In general, the antibiotics susceptibility profiles of the selected Enterobacteriaceae species showed the highest rate of susceptibility against Cefuroxime (94\%), Gentamicin (93\%), Ceftriaxone (91\%), Cefepime (89\%), Cefotaxime (85\%), Ceftazidime (84\%), Chloramphenicol (77\%), followed by Amoxicillin/Clavulanic acid and Ciprofloxacin (56\%) with least susceptibility identified in Ampicillin (46\%); Trimethoprim and Tetracycline (28\%) and sulfamethoxazole (17\%). The isolates were most resistance to Sulfamethoxazole (79\%), Trimethoprim (71\%), Tetracyclines (59\%), Ampicillin (49\%) and Amoxicillin/Clavunallic acid $(39 \%)$ respectively as shown in Table 2 below.

Table 1. Selected species of enterobacteriaceae isolated from commercial poultry production systems and Humans in Kiambu County.

\begin{tabular}{ccc}
\hline \multirow{2}{*}{ Bacteria species } & \multicolumn{2}{c}{ Bacteria isolated } \\
\cline { 2 - 3 } & Number & $\%$ \\
\hline E. coli & 289 & 48.9 \\
Klebsiella spps. & 83 & 14.0 \\
Salmonella spps. & 108 & 18.3 \\
Shigella spps. & 111 & 18.8 \\
Total & 591 & 100 \\
\hline
\end{tabular}


Table 2. Antimicrobial resistance profiles of 13 antibiotics agents tested against to the four selected Enterobacteriaceae of interest.

\begin{tabular}{lcccccc}
\hline Organisms & Antibiotic name & Number & \%R & \%I & \%S & \%R 95\% C.I. \\
\hline All organisms & Ampicillin & 591 & 49 & 5 & 46 & $45.1-53.3$ \\
All organisms & Amoxicillin/Clavulanic acid & 591 & 39 & 4 & 56 & $35.5-43.5$ \\
All organisms & Cefuroxime & 591 & 3 & 2 & 94 & $2.0-5.1$ \\
All organisms & Ceftazidime & 591 & 5 & 10 & 84 & $3.8-7.6$ \\
All organisms & Ceftriaxone & 591 & 4 & 5 & 91 & $2.8-6.3$ \\
All organisms & Cefotaxime & 591 & 7 & 8 & 85 & $4.9-9.2$ \\
All organisms & Cefepime & 591 & 3 & 7 & 89 & $2.1-5.3$ \\
All organisms & Gentamicin & 590 & 4 & 2 & 93 & $3.0-6.5$ \\
All organisms & Ciprofloxacin & 590 & 17 & 27 & 56 & $13.7-19.9$ \\
All organisms & Sulfamethoxazole & 589 & 79 & 4 & 17 & $75.0-81.8$ \\
All organisms & Trimethoprim & 590 & 71 & 1 & 28 & $67.5-74.9$ \\
All organisms & Chloramphenicol & 591 & 15 & 8 & 77 & $12.2-18.1$ \\
All organisms & Tetracycline & 591 & 59 & 13 & 28 & $55.1-63.2$ \\
\hline
\end{tabular}

Key: \%-p; R-resistance; I-intermediate; S-susceptibility; CI-confidence interval.

Table 3 presents the results of antimicrobial resistance profile of the 591 bacterial isolates tested against 13 antibiotics. Most the isolates were resistance to sulfamethoxazole (SMX), Trimethoprim (TMP), Tetracycline's (TCY) and Ampicillin (AMP) as show in Table 3 below.

Figure 1 presents percent distribution of resistance to the 13 tested antibiotics against the isolates. The figure indicates majority of the isolates we susceptible to Ceftazidime and Cefotaxime. However, most of isolates were resistance to Sulfamethoxazole, Trimethoprim, Tetracycline's and Ampicillin respectively.

Figure 2 presents the results of \% resistance by $E$. coli to the 13 tested antibiotics. Majority of the E. coli isolates were resistance to Sulfamethoxazole, Trimethoprim, Tetracycline, Ampicillin and Amoxi-Clavunalic acid, consecutively as displayed in Figure 2 below.

Figure 3 presents the results of \% resistance Klebsiella spps. to the 13 tested antibiotics. Majority of the Klebsiella spps. isolates were resistance to Sulfamethoxazole, Trimethoprim, Tetracycline, and Ampicillin consecutively as displayed in Figure 3 below.

Figure 4 presents the results of \% resistance Salmonella spps. to the 13 tested antibiotics. Majority of the Salmonella spps. were resistance to Tetracycline, Sulfamethoxazole, Trimethoprim, and Ampicillin Respectively as displayed in Figure 4 below.

Figure 5 presents the results of \% resistance Shigella spps. to the 13 tested antibiotics. Majority of the Shigella spps. were resistance to Amoxi-Clavunallic acid (AMC), Sulfamethoxazole (SMX), Trimethoprim (TMP), Ampicillin (AMP) and Tetracycline (TCY) respectively as displayed in Figure 5 below. 
Table 3. Antimicrobial resistance in various bacterial isolates $(n=591)$.

\begin{tabular}{|c|c|c|c|c|}
\hline Antimicrobial agent & $\begin{array}{l}\text { Escherichia coli } \\
\qquad(n=289)\end{array}$ & $\begin{array}{c}\text { Klebsiella sp. } \\
\quad(n=83\end{array}$ & $\begin{array}{l}\text { Salmonella sp. } \\
\quad(n=108\end{array}$ & $\begin{array}{c}\text { Shigella sp. } \\
(n=111)\end{array}$ \\
\hline AMC \%R & 45 & 2 & 7 & 84 \\
\hline AMP \%R & 48 & 60 & 39 & 55 \\
\hline CAZ \%R & 4 & 6 & 6 & 8 \\
\hline CHL \%R & 15 & 11 & 24 & 10 \\
\hline CIP \%R & 20 & 11 & 16 & 13 \\
\hline CRO \%R & 5 & 6 & 2 & 5 \\
\hline CTX \%R & 7 & 4 & 7 & 7 \\
\hline $\mathrm{CXM} \% \mathrm{R}$ & 3 & 4 & 1 & 5 \\
\hline FEP \%R & 2 & 5 & 3 & 5 \\
\hline GEN \%R & 4 & 5 & 6 & 4 \\
\hline SMX \%R & 82 & 72 & 77 & 77 \\
\hline TCY \% R & 56 & 58 & 82 & 48 \\
\hline TMP \%R & 78 & 60 & 67 & 68 \\
\hline
\end{tabular}

Key: R-Resistance; \%-Percentage; $\mathrm{n}-$ sample size; AMC-Amoxi-Clavunallic; AMP-Ampicillin, CAZ-Ceftazidime; CHL-Chloramphenicol; CIPCiprofloxacin; CRO—Ceftriaxone; CTX—Cefotaxime; CXM—Cefuroxime; FEP—Cefepime; GEN—Gentamicin; SMX—sulfamethoxazole; TCY—Tetracycline; TMP-Trimethoprim.

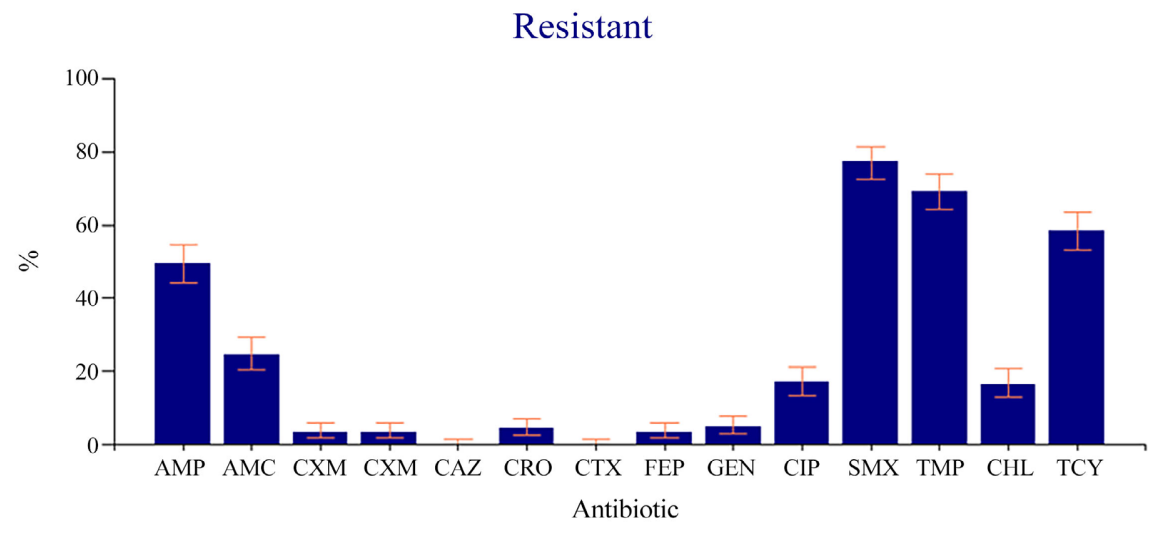

Figure 1. It presents percent distribution of resistance to the 13 tested antibiotics against the isolates.

eco

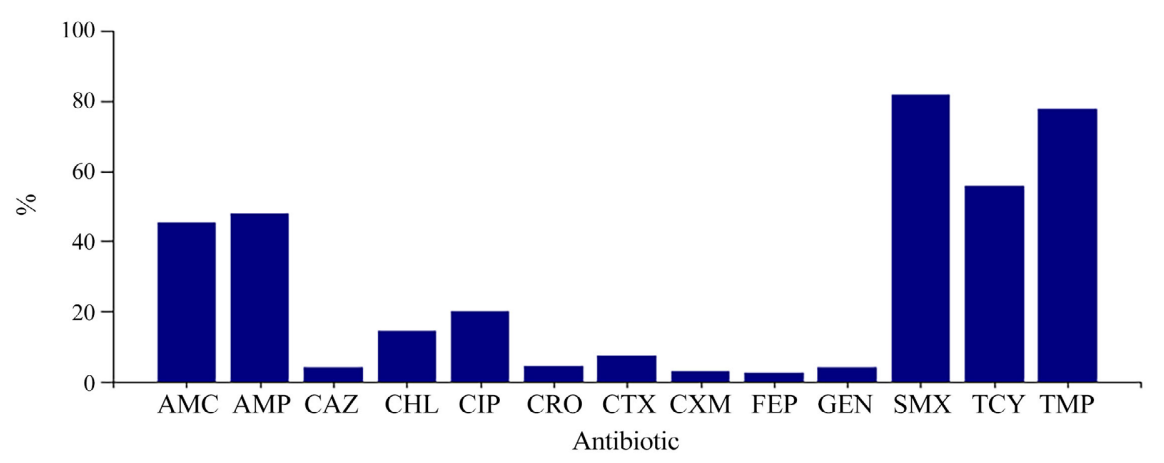

Figure 2. It presents the results of $\%$ resistance by E. coli to the 13 tested antibiotics. 
$\mathrm{k} 1-$

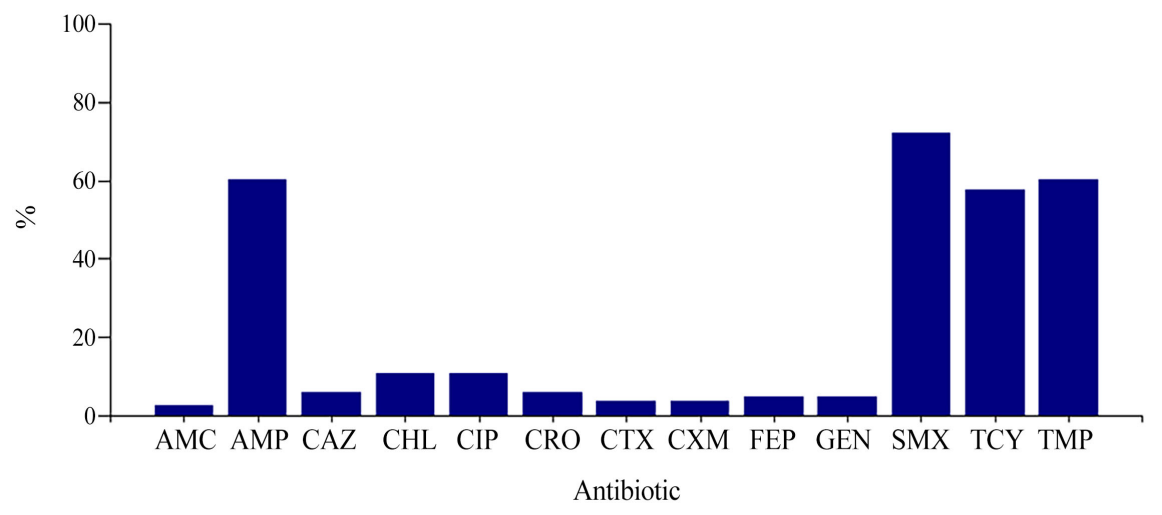

Figure 3. It presents the results of \% resistance Klebsiella spps. to the 13 tested antibiotics.

sal

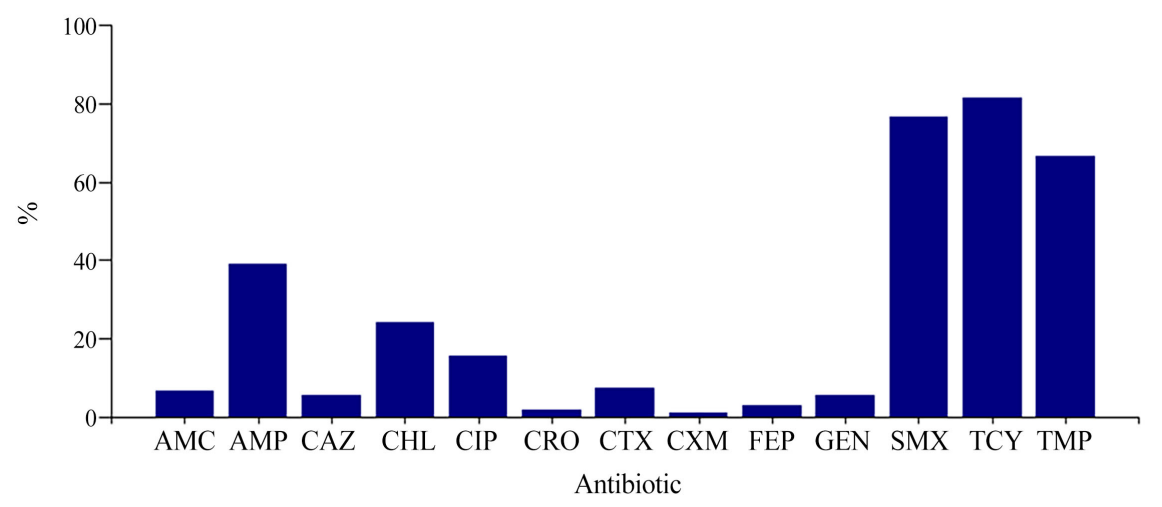

Figure 4. It presents the results of \% resistance Salmonella spps. to the 13 tested antibiotics.

shi

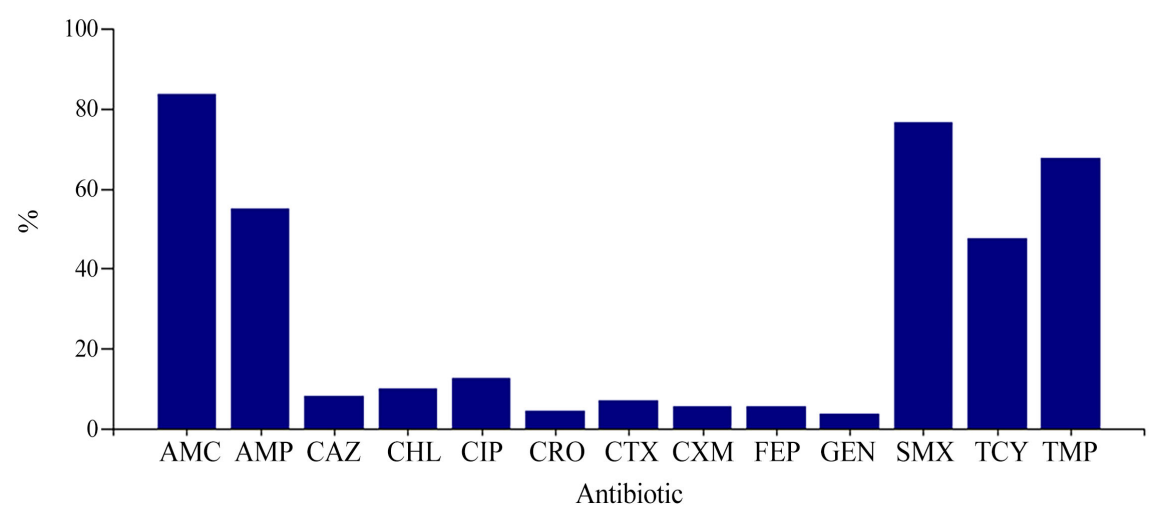

Figure 5. It presents the results of $\%$ resistance Shigella spps. to the 13 tested antibiotics.

Table 4 presents the results of the distribution of the isolates according to the species, source of the sample and their antibiotic resistance profiles. From the results we found that majority of the isolates across the four species of interest were from Layers and Improved Kienyeji birds. Furthermore, majority of the $E$. coli $(n=125)$ isolates were from layer birds followed by Improved Kienyeji $(n=$ 
92), Broiler birds $(n=55)$ and Broiler farmer $(n=17)$, respectively. We further, noted that most of Klebsiella spps. isolates emanated from Improved Kienyeji ( $n$ $=27)$, Broiler birds $(n=23)$, layer birds $(n=22)$ and Broiler farmer $(n=11)$, consecutively. For the Salmonella spps. majority of the isolates were from Layer birds $(n=45)$, followed by Improved Kienyeji $(n=31)$, Broiler birds $(n=26$ and Broiler farmer $(n=6)$. For the Shigella spps. isolates most were from layers $(n=$ $39)$ and Improved Kienyeji $(n=31)$ birds, with broiler bird having $(n=24)$ and Broiler farmer $(n=13)$, respectively. Majority of the E. coli, Salmonella spps., Klebsiella spps. and Shigella spps. Isolates were resistance to Sulfamethoxazole, Trimethoprim, Tetracycline's, and Ampicillin respectively. Both E. coli, and Shigella spps. also displayed great resistance to Amoxi-Clavunallic acid as shown in Table 4 below.

Table 5 presents the results of the distribution of the isolates according to the species, region of the sample collection and their antibiotic resistance profiles. The results indicates that most of the E. coli spps. came from Gatundu South ( $n$ $=60)$, Juja $(n=51)$, followed by Thika and Gatundu north $(n=48)$, respectively. We also noted that most of the Shigella spps. came from Juja $(n=41)$, with Salmonella spps. coming from Ruiru $(n=19)$. Majority of the Klebsiella spps. were isolated from Ruiru $(n=19)$, and Thika $(n=16)$, accordingly. We found that

Table 4. Antimicrobial resistance of bacterial isolates from the various sample types $(n=491)$.

\begin{tabular}{|c|c|c|c|c|c|c|c|c|c|c|c|c|c|c|c|}
\hline Organism & $\begin{array}{l}\text { Local specimen } \\
\text { code }\end{array}$ & $\begin{array}{c}\text { Number of } \\
\text { isolates }\end{array}$ & $\begin{array}{l}\mathrm{AMC} \\
\% \mathrm{R}\end{array}$ & $\begin{array}{c}\text { AMP } \\
\% R\end{array}$ & $\begin{array}{l}\text { CAZ } \\
\% R\end{array}$ & $\begin{array}{l}\mathrm{CHL} \\
\% \mathrm{R}\end{array}$ & $\begin{array}{l}\text { CIP } \\
\% R\end{array}$ & $\begin{array}{l}\text { CRO } \\
\% R\end{array}$ & $\begin{array}{l}\text { CTX } \\
\% R\end{array}$ & $\begin{array}{l}\text { CXM } \\
\% R\end{array}$ & $\begin{array}{l}\text { FEP } \\
\% R\end{array}$ & $\begin{array}{l}\text { GEN } \\
\% R\end{array}$ & $\begin{array}{l}\operatorname{SMX} \\
\% \mathrm{R}\end{array}$ & $\begin{array}{l}\text { TCY } \\
\% \mathrm{R}\end{array}$ & $\begin{array}{c}\text { TMP } \\
\% R\end{array}$ \\
\hline \multirow{4}{*}{ Escherichia coli } & Broiler & 55 & 29 & 56 & 6 & 15 & 29 & 7 & 7 & 6 & 4 & 2 & 87 & 56 & 80 \\
\hline & Broiler farmer & 17 & 59 & 53 & 12 & 18 & 18 & 12 & 12 & 12 & 6 & 6 & 94 & 41 & 88 \\
\hline & Improved Kienyeji & 92 & 39 & 49 & 5 & 15 & 19 & 5 & 8 & 2 & 3 & 5 & 84 & 59 & 82 \\
\hline & Layers & 125 & 55 & 42 & 2 & 14 & 18 & 2 & 6 & 2 & 1 & 4 & 77 & 55 & 72 \\
\hline \multirow{4}{*}{ Klebsiella sp. } & Broiler & 23 & 9 & 70 & 13 & 22 & 26 & 9 & 9 & 13 & 17 & 13 & 87 & 61 & 78 \\
\hline & Broiler farmer & 11 & 0 & 64 & 9 & 0 & 0 & 18 & 0 & 0 & 0 & 9 & 36 & 46 & 36 \\
\hline & Improved Kienyeji & 27 & 0 & 52 & 4 & 11 & 4 & 4 & 4 & 0 & 0 & 0 & 67 & 63 & 56 \\
\hline & Layers & 22 & 0 & 59 & 0 & 5 & 9 & 0 & 0 & 0 & 0 & 0 & 82 & 55 & 59 \\
\hline \multirow{4}{*}{ Salmonella $s p$} & Broiler & 26 & 0 & 46 & 4 & 23 & 8 & 0 & 8 & 4 & 4 & 4 & 72 & 81 & 73 \\
\hline & Broiler farmer & 6 & 0 & 50 & 17 & 17 & 17 & 0 & 0 & 0 & 0 & 0 & 50 & 50 & 50 \\
\hline & Improved Kienyeji & 31 & 10 & 39 & 7 & 36 & 19 & 3 & 10 & 0 & 3 & 3 & 87 & 87 & 68 \\
\hline & Layers & 45 & 9 & 33 & 4 & 18 & 18 & 2 & 7 & 0 & 2 & 9 & 76 & 82 & 64 \\
\hline \multirow{4}{*}{ Shigella sp. } & Broiler & 24 & 92 & 58 & 13 & 17 & 29 & 13 & 8 & 4 & 4 & 0 & 83 & 54 & 79 \\
\hline & Broiler farmer & 13 & 85 & 46 & 15 & 15 & 8 & 8 & 15 & 15 & 15 & 8 & 69 & 31 & 62 \\
\hline & Improved Kienyeji & 35 & 77 & 57 & 9 & 3 & 11 & 3 & 9 & 9 & 6 & 3 & 86 & 54 & 69 \\
\hline & Layers & 39 & 85 & 54 & 3 & 10 & 5 & 0 & 3 & 0 & 3 & 5 & 67 & 44 & 62 \\
\hline
\end{tabular}

Key: R-Resistance; \%-Percentage; $\mathrm{n}$-sample size; AMC-Amoxi-Clavunallic; AMP-Ampicillin, CAZ—Ceftazidime; CHL-Chloramphenicol; CIP_Ciprofloxacin; CRO-Ceftriaxone; CTX—Cefotaxime; CXM-Cefuroxime; FEP—Cefepime; GEN-Gentamicin; SMX—sulfamethoxazole; TCY—Tetracycline; TMP-Trimethoprim. 
Table 5. Antimicrobial resistance profiles of bacteria isolates tested against antimicrobial agents as per Sub-counties in which isolates were collected.

\begin{tabular}{|c|c|c|c|c|c|c|c|c|c|c|c|c|c|c|c|}
\hline Organism & Location & $\begin{array}{c}\text { Number of } \\
\text { isolates }\end{array}$ & $\begin{array}{c}\text { AMC } \\
\% R\end{array}$ & $\begin{array}{c}\text { AMP } \\
\% R\end{array}$ & $\begin{array}{c}\text { CAZ } \\
\% R\end{array}$ & $\begin{array}{c}\text { CHL } \\
\% \mathrm{R}\end{array}$ & $\begin{array}{l}\text { CIP } \\
\% R\end{array}$ & $\begin{array}{c}\text { CRO } \\
\% R\end{array}$ & $\begin{array}{c}\text { CTX } \\
\% R\end{array}$ & $\begin{array}{c}\text { CXM } \\
\% R\end{array}$ & $\begin{array}{l}\text { FEP } \\
\% \mathrm{R}\end{array}$ & $\begin{array}{l}\text { GEN } \\
\% \mathrm{R}\end{array}$ & $\begin{array}{c}\text { SMX } \\
\% R\end{array}$ & $\begin{array}{l}\text { TCY } \\
\% R\end{array}$ & $\begin{array}{c}\text { TMP } \\
\% R\end{array}$ \\
\hline \multirow{6}{*}{ E. coli } & Gatundu North & 48 & 71 & 42 & 2 & 17 & 25 & 4 & 13 & 2 & 2 & 6 & 75 & 67 & 75 \\
\hline & Gatundu south & 60 & 63 & 47 & 3 & 12 & 15 & 2 & 5 & 2 & 3 & 7 & 80 & 53 & 75 \\
\hline & Juja & 51 & 12 & 47 & 10 & 16 & 16 & 12 & 12 & 8 & 6 & 4 & 75 & 35 & 67 \\
\hline & Kikuyu & 39 & 49 & 59 & 3 & 23 & 18 & 3 & 5 & 3 & 0 & 0 & 90 & 51 & 84 \\
\hline & Ruiru & 43 & 9 & 49 & 2 & 14 & 28 & 5 & 2 & 2 & 2 & 2 & 95 & 58 & 86 \\
\hline & Thika & 48 & 63 & 46 & 4 & 8 & 21 & 2 & 6 & 2 & 0 & 4 & 81 & 71 & 83 \\
\hline \multirow{6}{*}{ Kleb. sp. } & Gatundu North & 9 & 0 & 56 & 0 & 0 & 0 & 0 & 0 & 0 & 0 & 0 & 78 & 78 & 89 \\
\hline & Gatundu south & 11 & 0 & 100 & 18 & 18 & 18 & 18 & 9 & 9 & 9 & 18 & 82 & 82 & 82 \\
\hline & Juja & 14 & 14 & 57 & 14 & 0 & 14 & 14 & 7 & 14 & 14 & 0 & 57 & 36 & 43 \\
\hline & Kikuyu & 14 & 0 & 43 & 0 & 36 & 7 & 7 & 0 & 0 & 0 & 0 & 86 & 64 & 50 \\
\hline & Ruiru & 19 & 0 & 53 & 5 & 5 & 16 & 0 & 0 & 0 & 5 & 11 & 53 & 37 & 47 \\
\hline & Thika & 16 & 0 & 63 & 0 & 6 & 6 & 0 & 6 & 0 & 0 & 0 & 88 & 69 & 69 \\
\hline \multirow{6}{*}{ Salmonella sp. } & Gatundu North & 10 & 10 & 30 & 10 & 20 & 30 & 0 & 0 & 0 & 0 & 0 & 70 & 90 & 70 \\
\hline & Gatundu south & 22 & 18 & 55 & 0 & 14 & 9 & 0 & 5 & 0 & 0 & 14 & 82 & 68 & 82 \\
\hline & Juja & 19 & 0 & 26 & 5 & 26 & 32 & 0 & 5 & 5 & 5 & 0 & 74 & 90 & 63 \\
\hline & Kikuyu & 26 & 4 & 35 & 4 & 27 & 15 & 0 & 15 & 0 & 4 & 4 & 73 & 77 & 54 \\
\hline & Ruiru & 7 & 0 & 71 & 0 & 29 & 14 & 0 & 0 & 0 & 0 & 14 & 100 & 100 & 86 \\
\hline & Thika & 24 & 4 & 33 & 13 & 29 & 4 & 8 & 8 & 0 & 4 & 4 & 75 & 83 & 63 \\
\hline \multirow{6}{*}{ Shigella sp. } & Gatundu North & 5 & 100 & 20 & 0 & 40 & 0 & 0 & 0 & 0 & 0 & 0 & 80 & 60 & 80 \\
\hline & Gatundu south & 12 & 83 & 50 & 8 & 8 & 17 & 0 & 8 & 8 & 8 & 17 & 75 & 50 & 67 \\
\hline & Juja & 41 & 95 & 56 & 15 & 7 & 22 & 10 & 10 & 5 & 7 & 0 & 78 & 49 & 76 \\
\hline & Kikuyu & 16 & 94 & 25 & 0 & 13 & 0 & 6 & 6 & 13 & 6 & 0 & 69 & 25 & 38 \\
\hline & Ruiru & 23 & 61 & 91 & 0 & 4 & 9 & 0 & 0 & 0 & 0 & 4 & 96 & 70 & 91 \\
\hline & Thika & 14 & 71 & 43 & 14 & 14 & 7 & 0 & 14 & 7 & 7 & 7 & 50 & 29 & 36 \\
\hline
\end{tabular}

Key: R-Resistance; \%-Percentage; $\mathrm{n}$-sample size; AMC-Amoxi-Clavunallic; AMP-Ampicillin, CAZ-Ceftazidime; CHL-Chloramphenicol; CIPCiprofloxacin; CRO-Ceftriaxone; CTX—Cefotaxime; CXM-Cefuroxime; FEP—Cefepime; GEN—Gentamicin; SMX—sulfamethoxazole; TCY—Tetracycline; TMP-Trimethoprim.

Klebsiella spps. from Gatundu South were 100\% resistance to Ampicillin as per Shigella spps. which had also $100 \%$ resistance to Amoxi-Clavunallic acid. Most of the isolates irrespective to area of origin had high resistance to Sulfamethoxazole, Trimethoprim and Tetracycline's, and Ampicillin respectively. Klebsiella spps., Shigella spps., and Salmonella spps. from Gatundu North were all to Ceftriaxone (CRO), Cefotaxime (CTX), Gentamicin (GEN), Cefuroxime (CXM), and Cefepime (FEP), consecutively. We also noted that Klebsiella spps., Shigella spps., and Salmonella spps. from Ruiru we all susceptible to Ceftriaxone (CRO), Cefotaxime (CTX), and Cefuroxime (CXM) respectively as indicated in Table 5. 


\section{Discussion}

Ever since the discovery of antimicrobial/antibiotics agents over eight decades ago, antibiotics have saved countless lives from infectious diseases and transformed modern medical procedures, including surgery, organ transplant and cancer treatment. However, over the years, the slow but steady spread of antibiotics resistance-whereby bacteria turn antibiotics ineffective-threatens to undo these important gains and take the world back to a pre-antibiotic era. While a significant role in the spread of such resistance has been played by the growing use of antibiotics in the human health sector, in recent years there has been recognition of the problems arising from even greater use of these miracle drugs in food-animal production. The emergence of antimicrobial-resistant bacterial pathogens has become a major public health concern. The use of antimicrobials in any venue, including disease treatment and growth promotion in domestic livestock, can potentially lead to widespread dissemination of antimicrobial-resistant bacteria [28].

The occurrence of multi-antimicrobial resistant Enterobacteriaceae poses a serious threat to the healthcare and Livestock Production system in Kenya as these organisms can spread from the environment to the hospital setting leading to nosocomial infections. In current study we determined the antimicrobial resistance patterns of selected enterobacteriaceae isolated from commercial poultry production systems and humans in Kiambu County, Kenya and their extent of distribution among the representative sub-counties.

Overall in the study found that majority of the isolates were E. coli $(48.9 \%)$, followed by Shigella spps. (18.8\%), consequently. This agrees with the study by Buxton and Frazer [29], and Bebora [30], that E. coli is the most common bacteria in animal and human fecal samples. This is further supported by a study that was carried by Njagi [31], who found $E$. coli prevalence of $40.2 \%$. In this study we demonstrates that there is high occurrence of single and multi-drug resistance (MDR) among the 13 antibiotics tested across the four enteric's. This resistance was highest among, Sulfamethoxazole (79\%), Trimethoprim (71\%), Tetracyclines (59\%), Ampicillin (49\%) and Amoxicillin/Clavunallic acid (39\%). This agrees with a study carried by Deng [32], and Nyabudi et al. [33], who found this antibiotics to be most commonly used in poultry production and to have developed resistance. Similarly, this has been demonstrated by other research such as: Allorechtova et al. [34]; Gakuya et al. [35]; Ombui et al. [36]. This call for joint effort for fight against MDR and X-DR by advocating prudent use of antimicrobial agent in animal production. However, the four enteric's were also highly susceptible to the following antibiotic; Cefuroxime (94\%), Gentamicin (93\%), Ceftriaxone (91\%), Cefepime (89\%), Cefotaxime (85\%), Ceftazidime (84\%), Chloramphenicol (77\%), followed by Amoxicillin/Clavulanic acid and Ciprofloxacin (56\%) respectively.

In addition, it was found that both E. coli, and Shigella spps. isolates displayed a great resistance to Amoxi-Clavunallic acid as shown in Table 4. The results 
also indicates that most of the E. coli spps. came from Gatundu South, Juja followed by Thika and Gatundu north, respectively. We also noted that most of the Shigella spps. came from Juja, with Salmonella spps. coming from Ruiru. Majority of the Klebsiella spps. were isolated from Ruiru and Thika accordingly. We found that Klebsiella spps. from Gatundu South were 100\% resistance to Ampicillin as per Shigella spps. which had also $100 \%$ resistance to Amoxi-Clavunallic acid. Most of the isolates irrespective to area of origin had high resistance to Sulfamethoxazole, Trimethoprim and Tetracycline's, and Ampicillin respectively. These indicates that the bacteria could have been exposed to these antibiotics previously hence acting as a selective force for resistance. High resistance realized to tetracycline is in line with earlier studies carried by Ansari and Khartoon; Kariuki et al., [37] [38] who reported that tetracycline is one of the broad spectrum antibiotics that are available in feed supplements and its improper use led to the development of antibiotic resistance. This could be attributed to either the misuse of drugs in therapeutic treatment, sub therapeutic preventive measure as feed additives to promote growth or use of disinfectants [39]. Several reports by Al-Bahry et al. [40], have indicated that multiple resistances were more common than resistance to a single antibiotic. It is possible that genes responsible for these multiple resistances are carried on the same plasmid. For example, in gram negative organisms, resistance is frequently regulated by genes that are normally associated with large plasmids which are conjugative. These plasmids often carry antibiotic resistance gene, heavy metals resistance genes and/or other pathogenic factors such as toxins, hence the selection for any of these factors selects for the plasmid which contain them as reported by Tricia et al. [41]. High, medium and low molecular weight plasmids were recovered during the study.

It was further noted that Klebsiella spps., Shigella spps., and Salmonella spps. from Gatundu North were all resistance to Ceftriaxone (CRO), Cefotaxime (CTX), Gentamicin (GEN), Cefuroxime (CXM), and Cefepime (FEP), consecutively. This agrees with a study carried by Zahraei and Farashi [42] who found an association between emergency of flouroquinolones resistance zoonotic pathogens with subsequent use of approved veterinary Antimicrobial agents in livestock production. This indicates there is irrational use of this antibiotics in poultry production systems in Kenya. According to Amy et al. [43], they found that overuse and misuse of antibiotics in poultry production systems would lead to a growing concern on it contribution to high resistance to antibiotics by pathogenic bacteria. This can act as a possible source of resistant genes which can be transferred to human pathogens via conjugation. We also noted that Klebsiella spps., Shigella spps., and Salmonella spps. from Ruiru we all susceptible to Ceftriaxone (CRO), Cefotaxime (CTX), and Cefuroxime (CXM) respectively as indicated in Table 5.

From the results it was found that majority of the isolates across the four enteric species of interest were from Layers and Improved Kienyeji birds. We further found that majority of the $E$. coli isolates were from layer birds followed by Improved Kienyeji, Broiler birds and Broiler farmer respectively. This is because 
most of the farmers in this areas preferred keeping layers birds for eggs and for improved Kienyeji to supply both meat and eggs. Also majority of the farmers were hesitant and did not consent to provide a stool samples for culturing. We further, noted that most of Klebsiella spps. isolates emanated from Improved Kienyeji, and Broiler birds. For the Salmonella spps. majority of the isolates were from Layer birds and Improved Kienyeji. For the Shigella spps. isolates most were from layers) and Improved Kienyeji birds. This Enterobacteriaceae cause infection in the bloodstream, surgical sites, urinary tract infections (UTIs), and respiratory tract infections and also cause frequent infections in diseases such as cancer and diabetes. The readily and easily spread of MDR Enterobacteriaceae from contaminated animal food sources and contact surfaces makes it a public health concern as indicated by McEwen and Fedorka Cray [44].

Therefore, the current study vindicate the need for Continuous surveillance of antimicrobial resistance by Enterobacteriaceae spps. in order to estimate the frequency of infection, the risk of infection, cost and possible treatment options available for these multidrug resistant pathogens. In addition, it is imperative to ascertain the sources and the routes of contaminations coined to the patterns of spread of this drug-resistant Enterobacteriaceae in Kenya for a suitable understanding of the dynamics involved and measures to prevent outbreaks in the community.

\section{Conclusion}

The current study indicates the importance of understanding the mechanisms underlying antibiotics drugs resistance in Enterobacteriaceae isolated from commercial poultry production systems and Human from fecal materials to help gain insights into the pattern of the spread of these drug-resistant strains and the best treatment options to use in treatment of infections.

\section{Study Limitations}

- Most of the farmers were hesitant to give human fecal sample hence the low number compared to cloacal swabs.

- Due to COVID-19 restriction of movements some of homes were not accessible for sample collection.

- We only did 13 antibiotic, and mostly targeted beta-Lactams, hence not all classes like aminoglycosides were included in the study.

\section{Acknowledgements}

The authors of this manuscript express their gratitude to everyone who contributed immensely to this research. Special thanks are due to Anita Chepkomei, Katana and Sharon Chepkurui for their tireless contributions and commitment during Lab work research assistance. The authors also register thanks to Prof. Samuel Kariuki, Acting Executive Director (KEMRI), for his guidance and material support in the conduct of this study. 


\section{Author's Contribution}

JGN, JKG, GOA and JMM conceptualized and designed the study. JGN conducted the study, collected data and performed data analysis. JGN, JKG, GOA, JMM, JM and SM interpreted the results and participated in the writing of the draft script, and affirmed that the manuscript was accurate and transparent account of the study being reported. JGN accept full responsibility for the work and the conduct of the study, and organized the decision to publish. The corresponding author confirms that all listed authors meet authorship criteria and no others meeting the criteria have been omitted.

\section{Ethical Approval}

Ethical approval was obtained from the faculty of veterinary medicine board of postgraduate study (UoN) and by NACOSTI (NACOSTI/P/21/8761). Permission to collected samples was sought from County Government of Kiambu Livestock, Fisheries and Veterinary Services (KCG/ALF/RESEARCH/VOL.1/49).

\section{Source of Funding}

The research study was partially funded by the National research fund-Kenya (NRF/PHD/02/166).

\section{Conflicts of Interest}

The authors declare no conflicts of interest regarding the publication of this paper.

\section{References}

[1] Jaciane, B.M., Pauline, C.B., Vanessa, A.A., Vanessa, C.F., Tanise, V.D., Grazielle, G.R., et al. (2015) Molecular Characterization of Enterobacteriaceae Resistant to Carbapenem Antimicrobials. Jornal Brasileiro de Patologia e Medicina Laboratorial, $51,162-165$.

[2] Bialvaei, A.Z. and Hossein Samadi, H.K. (2015) Colistin, Mechanisms and Prevalence of Resistance. Current Medical Research and Opinion, 31, 707-721. https://doi.org/10.1185/03007995.2015.1018989

[3] Luiz Mello Sampaio, J. and Cristina Gales, A. (2016) Antimicrobial Resistance in Enterobacteriaceae in Brazil: Focus on $\beta$-Lactams and Polymyxins. Brazilian Journal of Microbiology, 47, 31-37. https://doi.org/10.1016/j.bjm.2016.10.002

[4] John, N.K., Rohan, K., Collins, A., Musisi, N.L. and James, G.N. (2015) Phenotypic Expression and Prevalence of ESBL-Producing Enterobacteriaceae in Samples Collected from Patients in Various Wards of Mulago Hospital, Uganda. BMC Pharmacology and Toxicology, 16, Article No. 14. https://doi.org/10.1186/s40360-015-0013-1

[5] Rose, N., Beaudeau, F., Drouin, P., Toux, J.Y., Rose, V. and Colin, P. (1999) Risk Factors for Salmonella enterica subsp. Enterica Contamination in French Broiler-Chicken Flocks at the End of the Rearing Period. Preventive Veterinary Medicine, 39, 265-277. https://doi.org/10.1016/S0167-5877(99)00002-1

[6] Thriemer, K., Katuala, Y., Batoko, B., Alworonga, J.-P., Devlieger, H., Van Geet, C. 
and Jacobs, J. (2013) Antibiotic Prescribing in DR Congo: A Knowledge, Attitude and Practice Survey among Medical Doctors and Students. PLOS ONE, 8, Article ID: e55495. https://doi.org/10.1371/journal.pone.0055495

[7] Davies, J. and Davies, D. (2010) Origins and Evolution of Antibiotic Resistance. Microbiology and Molecular Biology Reviews, 74, 417-433.

https://doi.org/10.1128/MMBR.00016-10

[8] WHO Scientific Working Group (2014) Antimicrobial Resistance. Bulletin of the World Health Organization, 61, 383-394.

[9] FAO (Food and Agriculture Organization of the United Nations) (2002) Poultry Development Review. Food and Agriculture Organization of the United Nation, Rome. http://www.fao.org/3/i3531e/i3531e.pdf

[10] Moreki, J.C., Sentle, M.M., Chiripasi, S.C., Seabo, D. and Bagwasi, N. (2011) Prevalence of Diseases and Parasites of Rabbits in Botswana. Research Opinions in Animal and Veterinary Research, 1, 556-559.

[11] Morley, P.S., Apley, M.D., Besser, T.E., Burney, D.P., Fedorka-Cray, P.J., Papich, M.G., Traub-Dargatz, J.L. and Weese, J.S. (2005) Antimicrobial Drug Use in Veterinary Medicine. Journal of Veterinary Internal Medicine, 19, 617-629. https://doi.org/10.1111/j.1939-1676.2005.tb02739.x

[12] Landers, T.F., Cohen, B., Wittum, T.E. and Larson, E.L. (2012) A Review of Antibiotic Use in Food Animals: Perspective, Policy, and Potential. Public Health Reports, 127, 4-22. https://doi.org/10.1177/003335491212700103

[13] Muriuki, F.K., Ogara, W.O., Njeruh, F.M. and Mitema, E.S. (2001) Tetracycline Residue Levels in Cattle Meat from Nairobi Salughter House in Kenya. Journal of Veterinary Science, 2, 97-101. http://www.ncbi.nlm.nih.gov/pubmed/14614278

[14] Tharanya, S., Ghadhevaru, S., Dhinakar, R. and Preetha, P. (2018) Microbial Screening of Eggs for Detection of Antimicrobial Drug Residues-A Simple and Cost-Effective Method. International Journal of Advanced Biological Research, 8, 339-342.

[15] Nair, M.G., Guild, K.J., Du, Y., Zaph, C., Yancopoulos, G.D., Valenzuela, D.M., et al. (2008) Goblet Cell-Derived Resistinlike Molecule Beta Augments CD4 ${ }^{+}$T Cell Production of IFN-Gamma and Infection-Induced Intestinal Inflammation. The Journal of Immunology, 181, 4709-4715.

https://doi.org/10.4049/jimmunol.181.7.4709

[16] Carlet, J. (2012) The Gut Is the Epicentre of Antibiotic Resistance. Antimicrobial Resistance \& Infection Control, 1, Article No. 39. https://doi.org/10.1186/2047-2994-1-39

[17] European Centre for Disease Prevention and Control (n.d.) EARSS Net Database. http://ecdc.europa.eu

[18] Naylor, N.R., Rifat, A., Zhu, N., Kulasabanathan, K., Silva, S., Chatterjee, A., Knight, G.M. and Robotham, J.V. (2018) Estimating the Burden of Antimicrobial Resistance: A Systematic Literature Review. Antimicrobial Resistance and Infection Control, 7, Article No. 58. https://doi.org/10.1186/s13756-018-0336-y

[19] Cardona, C. and Kuney, D. (2002) Biosecurity on Chicken Farms. In: Bell, D.D. and Weaver, W.D., Eds., Commercial Chicken Meat and Egg Production, Springer, Boston, 543-556. https://doi.org/10.1007/978-1-4615-0811-3_28

[20] Nyaga, P. (2007) Poultry Sector Country Review (Kenya). Food and Agriculture Organization of the United Nations, Rome.

[21] Mugenda, O.M. and Mugenda, A.G. (2003) Research Methods: Quantitative and 
Qualitative Approaches. 2nd Edition, Act press, Nairobi.

[22] Bassetti, M., Merelli, M., Temperoni, C. and Astilean, A. (2013) New Antibiotics for Bad Bugs: Where Are We? Annals of Clinical Microbiology and Antimicrobials, 12, Article No. 22. https://doi.org/10.1186/1476-0711-12-22

[23] Clinical Laboratory Standard Institute (CLSI) (2013) Performance Standards for Antimicrobial Susceptibility Testing. 23rd Edition, M100-S23. Clinical Laboratory Standard Institute, Wayne.

[24] EL-Ganiny, A.M., EL-Mahdy, A.M., Abd EL-Latif, H.K., Ibrahem, R.H. and Abdelsabour, H.I. (2016) Phenotypic and Genotypic Detection of $\beta$-Lactams Resistance in Klebsiella Species from Egyptian Hospitals Revealed Carbapenem Resistance by OXA and NDM Genes. African Journal of Microbiology Research, 10, 339-347. https://doi.org/10.5897/AJMR2015.7871

[25] Koneman, E.W., Allen, S.D., Janda, W.M., Schreckenberger, P.C. and Winn, W.C. (1997) The Enterobacteriaceae. In: Koneman, E.W., Ed., Koneman's Color Atlas and Textbook of Diagnostic Microbiology, 5th Edition, Lippincott, 171-252.

[26] CLSI (Clinical Laboratory Standard Institute) (n.d.) Antimicrobial Susceptibility Testing (AST) Standards. https://clsi.org/education/microbiology/ast/

[27] Saidi, R., Khelef, D. and Kaidi, R. (2014) Antibiotic Susceptibility of Enterobacteraceae Species Isolated from Mastitic Milk in Algeria. Asian Pacific Journal of Reproduction, 3, 311-316. https://doi.org/10.1016/S2305-0500(14)60045-2

[28] Guerra, B., Soto, R., Helmuth, R. and Mendoza, M.C. (2002) Characterization of a Self-Transferable Plasmid from Salmonella enterica Serotype Typhimurium Clinical Isolates Carrying two Integron-Borne Gene Cassettes Together with Virulence and Drug Resistance Genes. Antimicrobial Agents and Chemotherapy, 46, 2977-2981. https://doi.org/10.1128/AAC.46.9.2977-2981.2002

[29] Buxton, A. and Frazer, B. (1977) Animal Microbiology. Blackwell Scientific Publications Ltd., Oxford/London.

[30] Bebora, L.C. (1979) A Study of the Occurrence of Avian Salmonellosis in Some Farms and a Slaughterhouse in Kenya. MSc. Thesis, University of Nairobi, Nairobi.

[31] Njagi, L.W. (2003) Carrier Status, Antibiotics and Disinfectant Sensitivity Patterns of Listeria monocytogenes and other Aerobi Bacteria in Scavenging Chicken and Ducks. MSc. Thesis, Universty of Nairobi, Nairobi.

[32] Deng, A.T.A. (2017) Status of Antimicrobial Resistance in Bacteria Isolated From Kenyan Chickens. University of Nairobi, Nairobi.

[33] Nyabundi, D., Onkoba, N.; Kimathi, R., Nyachieo, A., Juma, G., Kinyanjui, P. and Kamau, J (2017) Molecular Characterization and Antibiotic Resistance Profiles of Salmonella Isolated from Fecal Matter of Domestic Animals and Animal Products in Nairobi. Tropical Diseases, Travel Medicine and Vaccines, 3, Article No. 2. https://doi.org/10.1186/s40794-016-0045-6

[34] Allorechtova, K., Dolejska, M., Cizek, A., Tausova., Klimes, J., Bebora, L.C. and Literal, I. (2012) Dogs of Nomadic Pastroralists in Norther Kenya Are Reservoirs of Plasmid-Mediated Cephalosporins and Quinolone-Resistance Escherichia coli, Including Pandemic Clone B2-025-ST131. Journal of Antimicrobial Agents and Chemotherapy, 56, 4013-4017. https://doi.org/10.1128/AAC.05859-11

[35] Gakuya, F.M., Kyule, M.N., Gathura, P.B. and Kariuki, S.M. (2007) Antimicrobial Resistance of Bacterial Organisms Isolated from Rats. East African Medical Journal, 78, 646-649. https://doi.org/10.4314/eamj.v78i12.8934

[36] Ombui, J.N., Kimotho, A.M. and Nduhiu J.G. (2000) Antimicrobial Resistance Pat- 
terns and Plasmid Profiles of Staphylococcus aureus Isolated from Milk and Meat. East African Medical Journal, 77, 463-467. https://doi.org/10.4314/eamj.v77i9.46688

[37] Ansari, F.A. and Khatoon, H. (1999) A Survey of Antibiotic Resistance among $E$. coli Strains from Poultry in Karachi. Pakistan Journal of Pharmaceutical Sciences, 12, 7-14.

[38] Kariuki, S., Gilks, C., Kimari, J., Obanda, A., Muyodi J., Waiyaki, P. and Hart, C. (1999) Genotype Analysis of Escherichia coli Strains from Children and Chicken Living in Close Contact. Applied and Environmental Microbiology, 65, 472-476. https://doi.org/10.1128/AEM.65.2.472-476.1999

[39] Esipisup, I. (2009) Antibiotics Resistant Bug Found in Local Chicken. Daily Nation Tuesday, August 18, 2009.

[40] Al-Bahry, S.N., Al-Mashani, B.M., Elshafie, A.E., Pathare, N. and Al-Harthy, A.H. (2006) Plasmid Profile of Antibiotic Resistant E. coli Isolated from Chicken Intestines. Journal of the Alabama Academy of Science, 77, 152-159.

[41] Al-Bahry, S.N., Al-Mashani, B.M., Elshafie, A.E., Pathare, N. and Al-Harthy, A.H. (2006) Plasmid Profile of Antibiotic Resistant Escherichia Coli Isolated from Chicken Intestines. Journal of Alabama Academy of Sciences, 77, 152-159.

[42] Zahraei, T.S. and Farashi, S.B. (2006) Antibiotics Susceptibility Pattern of Escherichia coli Strains Isolated from Chickens with Colisepticemia in Tabriz Province, Iran. International Journal of Poultry Science, 5, 677-684.

https://doi.org/10.3923/ijps.2006.677.684

[43] Amy, R.S., Lisa, L., Shawn M. and Polly, W. (2007) What Do We Feed to Food Production Animals? A Review of Animal Feed Ingredients and Their Potential Impact on Human Health. Environmental Health Perspective, 115, 663-670. https://doi.org/10.1289/ehp.9760

[44] McEwen, S.A. and Fedorka-Cray P.J. (2002) Antimicrobial Use and Resistance in Animals. Clinical Infectious Diseases, 34, S93-S106. https://doi.org/10.1086/340246 